\title{
Anesthesia awareness. Can midazolam attenuate or prevent memory consolidation on intraoperative awakening during general anesthesia without increasing the risk of postoperative delirium?
}

There are numerous reviews on anesthesia awareness (AA) and post-traumatic stress disorder. Some authors assert that the use of benzodiazepines (BZDs) should be limited to patients requiring lower anesthetic drugs dosages for cardiac and emergency surgery, and for multiple-trauma patients. This is in agreement, at least in part, with the recommendations of the American Society of Anesthesiologists (ASA) [1]. Indeed, according to the ASA guidelines, the decision to administer BZDs should be made on a case-by-case basis, whereby the use of BZDs has been limited because of the risk of postoperative confusion and cognitive problems, including postoperative delirium (PD). This is one of the most common complications after major surgery, affecting $10-70 \%$ of surgical patients of 60 years and older. There are a number of factors associated with $\mathrm{PD}$, including hypoxia, hypercapnia, pain, stress, anxiety, fear, and psychotic and neurotic disorders, making it very difficult to determine the exact dose of midazolam - the BZD most used in anesthesia - that does not increase the risk of PD. Postoperative sedation using a midazolam drip $(0.5-2 \mathrm{mg} / \mathrm{h})$ results in delirium in $50 \%$ of the patients undergoing elective cardiac surgery.

Recently, there has been considerable interest in the adrenergic pathways involved in the mechanisms of consciousness and unconsciousness. Consequently, AA specialists propose the use of adrenergic agonists, including clonidine, as premedication. Others have suggested that dexmedetomidine can reduce the drug requirement during a bispectral index-guided anesthesia, with a lowered risk of delirium.

It is possibly wrong to condemn BZDs without a fair trial. The effect of BZDs on anterograde memory is well known. Therefore, when we use midazolam as a premedication, we protect the patient (or at least we try!) from the possibility that sensorial data can be consolidated into the long-term memory explicit or implicit - on the occurrence of intraoperative awakening during general anesthesia.

Indeed, AA should be prevented. However, what can we do when we realize that our patient has been exposed to a condition producing insufficient depth of hypnosis during general anesthesia, including interrupted administration of inhalation or intravenous anesthetics? The anterograde effect of BZDs can help us. However, what happens when we have not given midazolam (or another BZD) in premedication or when the event occurred long after midazolam administration, such that the drug no longer has an effect?

Several studies have suggested that the use of BZDs in anesthesia premedication may only have a limited effect on retrograde memory, and that sedation using this drug supplies partial anterograde amnesia without affecting retrograde memory [2]. In this regard, the retrograde facilitation (RF) phenomenon, a paradoxical event in which the recall of information presented before the administration of drugs is enhanced more than by the placebo, of midazolam and other BZDs is well known. However, because RF is an experimental condition, it is unclear whether this effect occurs in elderly individuals and whether it is influenced by plasma drug levels, baseline cognitive function, or genetic factors.

Interestingly, Timić et al. recently demonstrated the possibility of using these drugs to interfere with retrograde memory in an experimental study [3]. Previously, Semba et al. reported that midazolam (with propofol) provoked retrograde amnesia by increasing serotonergic transmission [4].

Based on these data, it is my opinion that we can use midazolam not only prophylactically in premedication, but also

(c) This is an open-access article distributed under the terms of the Creative Commons Attribution Non-Commercial License (http://creativecommons.org/ licenses/by-nc/3.0/), which permits unrestricted non-commercial use, distribution, and reproduction in any medium, provided the original work is properly cited. 
rapidly to attempt to prevent consolidation in the event of an unexpected emergence from surgical status during general anesthesia using clinical and instrumental hypnosis monitoring. This is particularly important when we consider that Dutton et al. [5] demonstrated that patients with very short episodes of intraoperative wakeful response were unlikely to form memories, whereas wakefulness of $>30$ seconds increased the risk of recall. Therefore, would 30 seconds be sufficient for a sensory stimulus to be stored in the explicit long-term memory, and thus be considered as an AA episode?

The main challenge is to not only find the appropriate midazolam dose to avoid the risk of AA, but also to prevent the induction of PD. We can only assume that there are a range of doses, whereby the effect on retrograde memory (when this can be demonstrated) occurs at different doses (higher or lower) than those interfering with the anterograde memory. Earlier sleep-laboratory studies on BZDs suggested that the amnesic effects depend on the dosage and type of substance. These ob- servations were confirmed by recent experimental studies demonstrating the complex mechanisms that link the areas involved in memory consolidation, including the hippocampus and substructures of the wider medial temporal lobe, and the rapidly working memory of the prefrontal cortex. This knowledge may allow the investigation of possible memory modulation during general anesthesia using several drugs. Although midazolam is a therapeutic choice, it should not be used at an arbitrary dosage. It would be of interest to investigate the effects of several midazolam doses on provoked recall during general anesthesia in an experimental rat model.

\section{Marco Cascella}

National Cancer Institute 'G Pascale’ Foundation of Naples, Via Mariano Semmola, Naples, Italy

E-mail: m.cascella@istitutotumori.na.it

\section{References}

1. American Society of Anesthesiologists Task Force on Intraoperative Awareness. Practice advisory for intraoperative awareness and brain function monitoring: a report by the American Society of Anesthesiologists Task Force on intraoperative awareness. Anesthesiology 2006; 104: 847-64.

2. Twersky RS, Hartung J, Berger BJ, McClain J, Beaton C. Midazolam enhances anterograde but not retrograde amnesia in pediatric patients. Anesthesiology 1993; 78: 51-5.

3. Timić T, Joksimović S, Milić M, Divljaković J, Batinić B, Savić MM. Midazolam impairs acquisition and retrieval, but not consolidation of reference memory in the Morris water maze. Behav Brain Res 2013; 241: 198-20.

4. Semba K, Adachi N, Arai T. Facilitation of serotonergic activity and amnesia in rats caused by intravenous anesthetics. Anesthesiology 2005; 102: 616-23.

5. Dutton RC, Smith WD, Smith NT. Brief wakeful response to command indicates wakefulness with suppression of memory formation during surgical anesthesia. J Clin Monit 1995; 11: 41-6. 


\section{In Response}

I am grateful to Dr. Marco Cascella for interest in my article on anesthesia awareness (AA). Dr. Marco Cascella suggestions are very interesting and should be helpful to the clinician. I agree that the appropriate benzodiazepine dose will help prevent AA from overwhelming benzodiazepine-induced delirium. However, it is unclear how much benzodiazepine should be administrated and which benzodiazepine would be the most useful and safe. Dr. Marco Cascella suggested midazolam as an appropriate benzodiazepine to prevent AA because it has an anterograde amnesia property. However, midazolam can cause postoperative delirium (PD). Thus, Dr. Marco Cascella suggested conducting an animal study to determine the appropriate midazolam dose. Midazolam is a relatively safe and economical drug. However, there are some limitations. First, PD is a serious condition even with the low prevalence [1]. Midazolam could trigger PD [2-4], which has high morbidity and mortality rates [5]. Thus, clinicians should not intentionally lead their patients into a risky situation. Second, an animal study differs from a patient. Many factors affect patient outcome, such as PD. Thus, it is difficult to apply animal study results to patients. It is encouraging to shed light on midazolam. However, midazolam may not be as attractive because of the considerable number of complications. Third, new agents are introduced frequently. Dexmetomidine is one of these new agents that prevents and relieves PD even in pediatric patients [6-9] and contributes to the prevention of AA. Dexmetomidine is an $\alpha_{2}$ agonist and effectively decreases AA if used as an adjuvant to general anesthesia because it acts on the central nervous system.

Therefore, I recommend further study using a new agent to prevent AA, even if a midazolam dose safe for preventing AA without PD can be titrated. This is a more favorable approach to clinicians and patients. Dexmedetomidine has limited published literature indicating that it prevents AA. Thus, I am interested in the optimal dexmedetomidine dose for preventing AA without side effects, such as bradycardia and delayed emergency, as well as the effectiveness of dexmedetomidine compared to conventional drugs such as benzodiazepines. Moreover, this is also an attractive strategy for managing delirium beyond PD [10].

\section{Hyun Sik Chung}

Department of Anesthesiology and Pain Medicine, Seoul St. Mary's Hospital, College of Medicine, The Catholic University of Korea, Seoul, Korea

E-mail: anesthe@catholic.ac.kr

\section{References}

1. Trabold B, Metterlein T. Postoperative delirium: risk factors, prevention, and treatment. J Cardiothorac Vasc Anesth 2014; $28: 1352-60$.

2. Pandharipande P, Cotton BA, Shintani A, Thompson J, Pun BT, Morris JA Jr, et al. Prevalence and risk factors for development of delirium in surgical and trauma intensive care unit patients. J Trauma 2008; 65: 34-41.

3. Santana Santos F, Wahlund LO, Varli F, Tadeu Velasco I, Eriksdotter Jonhagen M. Incidence, clinical features and subtypes of delirium in elderly patients treated for hip fractures. Dement Geriatr Cogn Disord 2005; 20: 231-7.

4. Carter GL, Dawson AH, Lopert R. Drug-induced delirium. Incidence, management and prevention. Drug Saf 1996; 15: 291-301.

5. Kim SD, Park SJ, Lee DH, Jee DL. Risk factors of morbidity and mortality following hip fracture surgery. Korean J Anesthesiol 2013; 64: 50510.

6. Dahmani S, Delivet H, Hilly J. Emergence delirium in children: an update. Curr Opin Anaesthesiol 2014; 27: 309-15.

7. Eremenko AA, Chernova EV. Treatment of delirium in the early postoperative period after cardiac surgery. Anesteziol Reanimatol 2014; (3): 30-4.

8. Sun Y, Lu Y, Huang Y, Jiang H. Is dexmedetomidine superior to midazolam as a premedication in children? A meta-analysis of randomized controlled trials. Paediatr Anaesth 2014; 24: 863-74.

9. Pasin L, Landoni G, Nardelli P, Belletti A, Di Prima AL, Taddeo D, et al. Dexmedetomidine reduces the risk of delirium, agitation and confusion in critically Ill patients: a meta-analysis of randomized controlled trials. J Cardiothorac Vasc Anesth 2014; 28: 1459-66.

10. Hilliard N, Brown S, Mitchinson S. A case report of dexmedetomidine used to treat intractable pain and delirium in a tertiary palliative care unit. Palliat Med 2015; 29: 278-81. 